\title{
Curvature and Uniformization
}

\author{
Rafe Mazzeo * \\ Stanford University \\ Michael Taylor ${ }^{\dagger}$ \\ University of North Carolina
}

\begin{abstract}
We approach the problem of uniformization of general Riemann surfaces through consideration of the curvature equation, and in particular the problem of constructing Poincaré metrics (i.e., complete metrics of constant negative curvature) by solving the equation $\Delta u-e^{2 u}=K_{0}(z)$ on general open surfaces. A few other topics are discussed, including boundary behavior of the conformal factor $e^{2 u}$ giving the Poincaré metric when the Riemann surface has smoothly bounded compact closure, and also a curvature equation proof of Koebe's disk theorem.
\end{abstract}

\section{Introduction}

Let $M$ be a smooth, connected, oriented two-dimensional manifold. A Riemannian metric $g$ on $M$ determines a conformal class

$$
[g]=\left\{e^{2 u} g: u \in C^{\infty}(M)\right\}
$$

and there is a well-known bijection between the set of conformal classes and the set of complex structures on $M$. A Riemann surface is such a surface endowed with a particular choice of conformal or complex structure.

It is reasonable to seek a canonical metric in each conformal class and a natural candidate is one with constant Gaussian curvature $K$. The case where $K$ is negative arises most frequently, and in any case is the one upon which we mostly concentrate. Thus we define a Poincaré metric on a Riemann surface $M$ to be one (in the conformal class of $M$ ) that is complete

\footnotetext{
${ }^{*}$ Research supported in part by NSF Grant DMS-9971975 and also at MSRI by NSF grant DMS-9701755. Email: mazzeo@math.stanford.edu

${ }^{\dagger}$ Research supported in part by NSF Grant DMS-9877077. Email: met@math.unc.edu
} 
and that has Gauss curvature $K \equiv-1$. A basic example is the Poincaré metric $G$ on the unit disk $D_{1} \subset \mathbb{R}^{2}$, which has components

$$
G_{j k}=\frac{4}{\left(1-r^{2}\right)^{2}} \delta_{j k}, \quad r^{2}=x_{1}^{2}+x_{2}^{2}
$$

This is the unique Poincaré metric in $[\delta]$, and it is invariant with respect to all conformal (or holomorphic) automorphisms of $D_{1}$.

If $g_{0}$ is a metric on $M$, with Gauss curvature function $K_{0}(x)$, then $g=e^{2 u} g_{0}$ has Gauss curvature $K=\left(K_{0}-\Delta u\right) e^{-2 u}$, which is equal to -1 provided $u$ satisfies

$$
\Delta u-e^{2 u}=K_{0}(x)
$$

In particular, to find a Poincaré metric $g \in\left[g_{0}\right]$ it is sufficient to solve (1.2) and show that $e^{2 u} g_{0}$ is complete.

Poincaré metrics are closely related to conformal (holomorphic) coverings by $D_{1}$. In fact, a Poincaré metric $g$ on $M$ lifts to a Poincaré metric $\tilde{g}$ on the universal cover $\widetilde{M}$, and the covering map $\varphi: \widetilde{M} \rightarrow M$ is by definition a local isometry, hence conformal. On the other hand, a basic theorem in differential geometry asserts that $(\widetilde{M}, \widetilde{g})$ is isometric to the disk $D_{1}$ with its Poincaré metric (1.1). Therefore we obtain a holomorphic covering map

$$
\varphi: D_{1} \longrightarrow M
$$

which is a local isometry between the Poincaré metrics on $D_{1}$ and $M$. Conversely, if $\varphi$ is any such conformal covering map, the deck transformations on $D_{1}$ are conformal and thus fix the Poincaré metric there. Hence $\varphi$ acts by isometries and pushes down to a Poincaré metric on $M$. Extending this reasoning slightly, we see that

If a Poincaré metric exists on a Riemann surface $M$, it is unique.

The discussion in the last paragraph makes it clear that the construction of Poincaré metrics is intimately related to the classical uniformization theorem, which we now state:

Uniformization Theorem Every simply-connected Riemann surface is holomorphically equivalent to either $\widehat{\mathbb{C}}, \mathbb{C}$, or $D_{1}$.

Here $\widehat{\mathbb{C}}$ denotes the Riemann sphere. An equivalent statement is that any (connected) Riemann surface $M$ can be holomorphically covered by $\widehat{\mathbb{C}}, \mathbb{C}$, or $D_{1}$. It is well known that this result can be established when $M$ is compact 
by directly solving the curvature equation; cf. $\S 8$ for further discussion of this. One of our goals here is to give a direct treatment of the curvature equation on a broad class of Riemann surfaces, and to use this to establish the uniformization theorem.

We proceed in a series of relatively easy steps. In $\S 2$ we commence by finding a Poincaré metric when $M$ is the interior of a compact smooth surface with boundary. Section 3 takes up another theme, the boundary behavior of the Poincaré metric in this case. In $\S 4$, an approximation argument is used to produce a Poincaré metric on any domain $\Omega$ in the complex plane whose complement has at least two points. In $\S 5$ we take the space to advertise a purely curvature proof of Koebe's disk theorem, and its well known corollary about normality of a family of univalent maps. Section 6 establishes the uniformization theorem for general simply connected Riemann surfaces, as a consequence of results of sections 2 and 5 . In $\S 7$ we relate the dichotomy between Riemann surfaces covered by $D_{1}$ and those covered by $\mathbb{C}$ to a dichotomy in the behavior of the curvature equation. In $\S 8$ we discuss the uniformization theorem for compact surfaces.

We say more about the second main theme of this paper, taken up in $\S 3$. Many developments in modern function theory have focused on the connection between the regularity of the boundary of $M$ (especially when it is a planar domain) and the regularity of the mapping $\varphi$. From the point of view here, it seems also of interest to examine the boundary behavior of the solution to (1.2) yielding the Poincaré metric, especially when $M$ has compact closure in a larger Riemann surface. The boundary regularity results we obtain in $\S 3$ on $e^{-u}$ have implications for the qualitative behavior of the covering map $D_{1} \rightarrow M$.

We conclude this introduction by providing a few explicit examples of Poincaré metrics to illustrate various phenomena that can occur. Also, we will have specific use for several of these formulas in $\S 3$ and $\S 4$.

- The upper half-plane $H^{+}=\left\{x \in \mathbb{R}^{2}: x_{2}>0\right\}$ has Poincaré metric

$$
g_{j k}=x_{2}^{-2} \delta_{j k} \text {. }
$$

This may be obtained from (1.1) using the standard linear fractional transformation that maps $D_{1}$ to $H^{+}$.

- The Poincaré metric on the punctured disk $D^{*}=\left\{x \in \mathbb{R}^{2}: 0<|x|<\right.$ $1\}$ is

$$
g_{j k}=\left(r \log \frac{1}{r}\right)^{-2} \delta_{j k},
$$


as can be verified using the covering $H^{+} \rightarrow D^{*}, z \mapsto e^{i z}$.

- The strip $\Sigma=\left\{x \in \mathbb{R}^{2}: 0<x_{2}<\pi\right\}$ has Poincaré metric

$$
g_{j k}=\left(\sin x_{2}\right)^{-2} \delta_{j k},
$$

as one obtains from (1.4) via the conformal diffeomorphism $\Sigma \rightarrow$ $H^{+}, z \mapsto e^{z}$.

- The annulus $A_{b}=\left\{x \in \mathbb{R}^{2}: e^{-\pi / b}<|x|<1\right\}$ has Poincaré metric

$$
g_{j k}=\left[\frac{b}{r \sin \left(b \log \frac{1}{r}\right)}\right]^{2} \delta_{j k},
$$

as can be seen using (1.6) and the covering $\Sigma \rightarrow A_{b}, z \mapsto e^{i z / b}$. Note that the $b \rightarrow 0$ limit gives (1.5).

- The quarter-plane $Q=\left\{x \in \mathbb{R}^{2}: x_{1}>0, x_{2}>0\right\}$ has Poincaré metric

$$
g_{j k}=\frac{x_{1}^{2}+x_{2}^{2}}{x_{1}^{2} x_{2}^{2}} \delta_{j k}
$$

as one obtains from (1.4) via the map $Q \rightarrow H^{+}, z \mapsto z^{2}$.

\section{Smoothly bounded Riemann surfaces}

Let $\bar{\Omega}$ be a compact, oriented, connected 2-dimensional Riemannian manifold with smooth boundary, with metric $g_{0}$. We can suppose that $\bar{\Omega}$ is contained in a larger open Riemann surface $M$. We produce a solution $u$ to (1.2) as a limit, and then show it is complete.

Given $a \in(0, \infty)$, the existence of a unique solution $u_{a} \in C^{\infty}(\bar{\Omega})$ to (1.2) with $u_{a}=a$ on $\partial \bar{\Omega}$ is easy and well known; cf. Chapter 14, $\S 1$ of [20]. The proof given there uses a simple combination of variational techniques and maximum principle arguments. Our strategy is to take $a \nearrow \infty$. Thus we need to consider how $u_{a}$ depends on $a$.

Lemma 2.1 These solutions are monotonic in the parameter a:

$$
a<b \Longrightarrow u_{a} \leq u_{b} \text { on } \Omega \text {. }
$$


Proof: Set $v=u_{b}-u_{a}$. Then $\left.v\right|_{\partial \Omega}=b-a>0$, while

$$
\Delta v-\varphi_{a b} v=0
$$

with

$$
\varphi_{a b}=\frac{e^{2 u_{b}}-e^{2 u_{a}}}{u_{b}-u_{a}}=\frac{1}{u_{b}-u_{a}} \int_{u_{a}}^{u_{b}} 2 e^{2 \sigma} d \sigma>0 .
$$

Say $v_{\min }=v(p), p \in \Omega$. If $v(p)<0$, then $\Delta v(p)=\varphi_{a b}(p) v(p)<0$, which is impossible, so $v \geq 0$ on $\Omega$, yielding (2.1).

Remark 2.1. This lemma and its variants will be used repeatedly in the following. In other useful variants, $a$ and $b$ can be functions on $\partial \Omega$, rather than just constants, and we can also compare functions $u_{a}$ and $u_{b}$ that satisfy

$$
\Delta u_{a}+k_{a} e^{2 u_{a}}=K_{0}, \quad \Delta u_{b}+k_{b} e^{2 u_{b}}=K_{0} .
$$

If $-k_{a} \geq-k_{b}>0$ on $\bar{\Omega}$, then (2.1) holds.

The next step is to obtain a uniform upper bound for this monotonic sequence.

Lemma 2.2 There exists a locally bounded function $B$ on $\Omega$ such that

$$
e^{2 u_{a}(p)} \leq B(p), \quad \forall a<\infty .
$$

Proof: First consider the case where $\Omega$ is a planar domain, $\Omega \subset \mathbb{R}^{2}$. Define $\delta(p)=\operatorname{dist}(p, \partial \Omega)$. We claim that

$$
e^{2 u_{a}(p)} \leq \frac{4}{\delta(p)^{2}}, \quad \forall a<\infty .
$$

In fact, for any $\beta \in(0, \delta(p))$, let $D_{\beta}(p)$ be the disk of radius $\beta$, centered at $p$, with its Poincaré metric

$$
g_{j k}=e^{2 w} \delta_{j k}=\frac{4 \beta^{2}}{\left(\beta^{2}-r^{2}\right)^{2}} \delta_{j k}, \quad r=|x-p| .
$$

Since $w$ solves (1.2) and tends to $+\infty$ on $\partial D_{\beta}(p)$, Lemma 2.1 gives

$$
u_{a} \leq w \text { on } D_{\beta}(p) .
$$


(2.6) follows as $\beta \nearrow \delta(p)$.

For the general case, use isothermal coordinates to get a neighbourhood $p \in \mathcal{O}_{p} \subset \Omega$ and a conformal map $\psi_{p}: D_{1} \rightarrow \mathcal{O}_{p}$. We may assume that $\partial \mathcal{O}_{p}$ is smooth and $\psi_{p}$ extends to a diffeomorphism on the closure. The Poincaré metric $g_{p}=e^{2 w_{p}} g_{0}$ on $\mathcal{O}_{p}$ yields a barrier, and hence an upper bound $w \leq B$, as above. It is clearly possible to choose $B$ as a continuous function.

Using Lemma 2.2, we now let $a \nearrow \infty$ and obtain

$$
u_{a}(p) \nearrow u(p), \quad \forall p \in \Omega, \quad e^{2 u(p)} \leq B(p) .
$$

Each derivative of $u_{a}$ is locally uniformly bounded by elliptic regularity, so convergence takes place in $C_{\mathrm{loc}}^{\infty}(\Omega)$, and hence $u$ is a solution of (1.2).

Remark 2.2. The upper bound (2.6), valid for the limit $u$, is not sharp as $p$ tends toward $\partial \Omega$. An only slightly more involved argument, using a conformal self-map of the disk $D_{\beta}(p)$, shows that when $\partial \Omega$ is smooth, $\delta(p)^{2} e^{2 u(p)} \rightarrow 1$ as $p \rightarrow \partial \Omega$. A more refined result along these lines is the content of Proposition 3.1 below.

It remains to demonstrate completeness.

Lemma 2.3 Assume $\bar{\Omega}$ is a smoothly bounded and compact surface with metric $g_{0}$, and $\Omega$ is its interior. If $u$ is given as above, as the limit of the $u_{a}$, then the corresponding metric $g=e^{2 u} g_{0}$ is complete on $\Omega$.

Proof: Let $\gamma:[0, L) \rightarrow \Omega$ be a unit-speed geodesic for $g$, with $L<\infty$, and suppose that $\gamma(t)$ does not converge to a point in $\Omega$ as $t \rightarrow L$. This curve also has finite length with respect to $g_{0}$, and so there exists $p \in \bar{\Omega}$ such that $\gamma(t) \rightarrow p$ as $t \rightarrow L$.

As before, first consider the case where $\Omega$ is planar. Let $\mathcal{D}_{p} \subset \mathbb{R}^{2} \backslash \Omega$ be a disk, tangent to $\partial \Omega$ at $p$. Regard $\mathbb{R}^{2}$ as sitting inside the Riemann sphere $\widehat{\mathbb{C}}$ and consider $\mathcal{D}_{p}^{\prime}=\widehat{\mathbb{C}} \backslash \mathcal{D}_{p}$, with its Poincaré metric $h$. Thus $\Omega \subset \mathcal{D}_{p}^{\prime}$. The argument used in the first part of the proof of Lemma 2.2, applied to a sequence of disks decreasing to $\mathcal{D}_{p}^{\prime}$, also gives

$$
g \geq h \text { on } \Omega .
$$

But $\left(\mathcal{D}_{p}^{\prime}, h\right)$ is complete, so the $h$-length of $\gamma$ is infinite, and hence $\Omega$ is complete with respect to $g$.

To handle the general case, assume that $\bar{\Omega}$ sits inside a slightly larger (open) Riemann surface $M$ and the metric $g_{0}$ is extended smoothly. If 
$\gamma(t) \rightarrow p \in \partial \Omega$ as $t \nearrow L$, choose a small holomorphic disk $\mathcal{D}$ containing $p$, roughly cut in half by $\partial \Omega$. Choose a smooth curve in $\mathcal{D} \backslash \bar{\Omega}$ hitting $\partial \Omega$ transversally at $p$ and let $p_{j} \rightarrow p$ along this curve. Denote by $e^{2 v_{j}} g_{0}$ the Poincaré metric on $\mathcal{D} \backslash\left\{p_{j}\right\}$ obtained by pulling back (1.5), and let $\mathcal{O} \subset \subset \mathcal{D}$ be a smaller disk containing $p$ and the sequence $p_{j}$. We see that, for each $j<\infty$, there exists $A(j)<\infty$ such that

$$
u_{a} \geq v_{j} \text { on } \partial \Omega \cap \mathcal{O}, \text { for } a \geq A(j) .
$$

Also, considering $u_{1}$ (which equals 1 on $\partial \Omega$ ) we see that there exists $B>0$ such that $u_{1} \geq v_{j}-B$ on $\partial \mathcal{O} \cap \Omega$, for all $j$, hence

$$
u_{a} \geq v_{j}-B \text { on } \partial \mathcal{O} \cap \Omega, \text { for } a \geq 1 .
$$

Hence, by Remark 2.1,

$$
u_{a} \geq v_{j}-B \text { on } \mathcal{O} \cap \Omega, \text { for } a \geq \max (1, A(j)),
$$

so

$$
u \geq v_{j}-B \text { on } \mathcal{O} \cap \Omega, \quad \forall j .
$$

Hence

$$
u \geq v-B \text { on } \mathcal{O} \cap \Omega,
$$

where $e^{2 v} g_{0}$ is (1.5) pulled back to $\mathcal{D} \backslash\{p\}$. This is enough to give completeness.

Putting these lemmas together we obtain

Proposition 2.4 If $\Omega$ is the interior of a smooth two-dimensional manifold with boundary $\bar{\Omega}$, then $\Omega$ admits a Poincaré metric.

As we have emphasized, Proposition 2.4 implies $\Omega$ is holomorphically covered by $D_{1}$. In particular, if $\Omega$ is simply connected then there exists a holomorphic diffeomorphism $\varphi: D_{1} \rightarrow \Omega$. It is useful to recall the linear PDE treatment of this result, in which one picks $p \in \Omega$ and takes the Green function $u \in H^{1-\varepsilon}(\Omega) \cap C^{\infty}(\bar{\Omega} \backslash\{p\})$, satisfying

$$
\Delta u=2 \pi \delta_{p}, \quad \text { on } \Omega,\left.\quad u\right|_{\partial \Omega}=0 .
$$

Then $u(x)$ behaves like $\log |x|$ in local normal coordinates centered at $p$, and the hypothesis that $\Omega$ is simply connected implies that there exists a 
harmonic conjugate $v$, smooth and well defined $\bmod 2 \pi \mathbb{Z}$, on $\bar{\Omega} \backslash\{p\}$, and the function

$$
\Phi(x)=e^{u(x)+i v(x)}
$$

yields a holomorphic map $\Phi: \Omega \rightarrow D_{1}$, extending to a smooth map $\Phi: \bar{\Omega} \rightarrow$ $\bar{D}_{1}$. It follows from the Hopf lemma (also known as Zaremba's principle) that $\partial_{\nu} u<0$ on $\partial \Omega$, which via the Cauchy-Riemann equations implies $\Phi$ maps $\partial \Omega$ locally diffeomorphically onto $S^{1}=\partial D_{1}$. Now the argument principle implies the degree of the map $\partial \Omega \rightarrow S^{1}$ equals the number of preimages of any $q \in D_{1}$ (counting multiplicity). This number is clearly one for $q=0$, so $\Phi$ is the desired holomorphic diffeomorphism. This argument gives us something extra; $\Phi$ extends to a smooth diffeomorphism of $\bar{\Omega}$ onto $\bar{D}_{1}$. This has implications for the boundary behavior of the Poincaré metric on $\Omega$, which we will explore and extend in the next section.

\section{Boundary regularity when $\bar{\Omega}$ is smooth}

In this section we analyze the boundary behavior of the function $u \in C^{\infty}(\Omega)$ providing the Poincaré metric $e^{2 u} g_{0}$, when $\Omega$ is smoothly bounded. Throughout this section we let $x$ denote the distance function (with respect to $g_{0}$ ), which is well-defined and smooth in some sufficiently small neighbourhood of the boundary $\partial \Omega$, and shall often also use $y$ as a local coordinate along $\partial \Omega$.

Proposition 3.1 Suppose that $\bar{\Omega}$ is smoothly bounded and compact, with Poincaré metric $e^{2 u} g_{0}$. Then as $x \rightarrow 0, u$ has an asymptotic expansion of the form

$$
u(x, y) \sim \log (1 / x)+u_{1}(y) x+u_{2}(y) x^{2}+\cdots,
$$

where the coefficient functions $u_{j}(y)$ all lie in $\mathcal{C}^{\infty}(\partial \Omega)$. Equivalently,

$$
e^{-u} \in C^{\infty}(\bar{\Omega}), \text { and } \partial_{\nu} e^{-u} \equiv 1 \text {. }
$$

This is the direct analogue of the expansion valid for solutions of the singular Yamabe problem in higher dimensions (at least in the most favourable case), cf. [15]. The proof has two steps: first, barrier techniques are used to obtain rough (scale-invariant) estimates for the solution, and at that point 
some techniques from the linear analysis of [14 are used to improve this to full tangential regularity and an expansion.

Remark 3.1. We note that the second condition in (3.2) is an automatic consequence of the first. In fact, $W=e^{-u}$ satisfies

$$
\Delta W=\frac{|\nabla W|^{2}-1}{W}-K_{0} W,\left.\quad W\right|_{\partial \Omega}=0 .
$$

If $W \in C^{\infty}(\bar{\Omega})$, then the right side of (3.3) must be continuous on $\bar{\Omega}$, which implies $\left.\partial_{\nu} W\right|_{\partial \Omega}=1$.

Remark 3.2. In case $\bar{\Omega}$ is smoothly bounded and simply connected, the smoothness of $e^{-u}$ on $\bar{\Omega}$ is a simple consequence of the fact that the holomorphic diffeomorphism $\Phi: \Omega \rightarrow D_{1}$ given by (2.17) extends to a smooth diffeomorphism $\Phi: \bar{\Omega} \rightarrow \bar{D}_{1}$, plus the fact that $e^{2 u} g_{0}=\Phi^{*}\left(g_{P}\right)$, where $g_{P}$ is the Poincaré metric on $D_{1}$.

Remark 3.3. The smoothness condition in (3.2) is clearly invariant when $g_{0}$ is replaced by $g_{1}=e^{2 w} g_{0}$ with $w \in C^{\infty}(\bar{\Omega})$, and hence so are the rest of the conclusions in Proposition 3.1.

We will implement Remark 3.3 using the following result.

Lemma 3.2 For each connected component $\gamma$ of $\partial \Omega$, there is a collar neighborhood $\mathcal{C}$ and $a C^{\infty}$ conformal diffeomorphism $\varphi: \overline{\mathcal{C}} \rightarrow \bar{A}_{b}$ onto an annulus $\bar{A}_{b}=\left\{z \in \mathbb{C}: e^{-\pi / b} \leq|z| \leq 1\right\}$, as in (1.7).

Proof: Taking a collar neighborhood $\mathcal{C}_{0}$ of $\gamma$, we can produce a simply connected $\mathcal{O}$ with smooth boundary such that a collar neighborhood of $\partial \mathcal{O}$ is identified with $\mathcal{C}_{0}$. Then we can apply the construction mentioned at the end of $\S 2$, obtaining a $C^{\infty}$ conformal diffeomorphism $\Phi: \overline{\mathcal{O}} \rightarrow \bar{D}_{1}$. The inverse image of $\bar{A}_{b}$, for $b$ sufficiently large, can then be identified with the desired collar neighborhood $\overline{\mathcal{C}}$ of $\gamma$.

Using Lemma 3.2, we can construct $w \in C^{\infty}(\bar{\Omega})$ such that $g_{1}=e^{2 w} g_{0}$ has the property that each boundary component $\gamma$ of $\partial \Omega$ has a collar neighborhood that is isometric to $\bar{A}_{b}$. We now renotate, giving $g_{1}$ the label $g_{0}$. We are ready to establish the following estimate. 
Lemma 3.3 In the setting of Proposition 3.1, we have, near $\partial \Omega$,

$$
u=\log \frac{1}{x}+v, \quad|v| \leq C x .
$$

Proof: Recall the conformal diffeomorphism $\varphi: \overline{\mathcal{C}} \rightarrow \bar{A}_{b}$ constructed in Lemma 3.2. Pulling the Poincaré metric (1.7) on $A_{b}$ back via $\varphi$ produces the Poincaré metric on $\mathcal{C}$, say $e^{2 u_{1}} g_{0}$, and we clearly have

$$
u \leq u_{1} \text {, on } \mathcal{C} \text {. }
$$

It is clear from the representation (1.7) that this produces an upper bound on $u$ of the form asserted in (3.4).

It remains to produce an appropriate lower bound on $\left.u\right|_{\mathcal{C}}$. It is equivalent to produce a lower bound on the metric $\left.e^{2 u} g_{0}\right|_{\mathcal{C}}$, pulled back to $A_{b}$ via $\varphi$. To accomplish this, we supplement the family of metrics (1.7) with the following family of metrics on $A_{b}$ :

$$
g_{j k}^{\beta}=\left[\frac{\beta}{r \sinh \left(\beta \log \frac{1}{r}\right)}\right]^{2} \delta_{j k} .
$$

These are obtained by analytically continuing (1.7) to purely imaginary $b$. A direct check shows that these metrics satisfy (1.2) on $\left\{x \in \mathbb{R}^{2}: 0<|x|<1\right\}$, for each $\beta \in(0, \infty)$, and the metric (1.5) arises as the limit as $\beta \searrow 0$. The metrics (3.6) are complete at the outer boundary $\{z:|z|=1\}$, and, given any fixed $b>0$, for large $\beta$ they are quite small on the inner boundary $\{z:|z|=b\}$ of $A_{b}$. Choosing $\beta$ sufficiently small gives the desired lower bound, establishing (3.4).

What we have accomplished thus far is to show that the conformal factor $u$ giving the Poincaré metric $g=e^{2 u} g_{0}$ may be written on a collar neighborhood $\mathcal{C}$ of each boundary component as $u=\log (1 / x)+v$, where $|v| \leq C x$ for $0<x \leq x_{0}$. Here $x=1-r, r=|z|$ on the annulus $A_{b}$, identified with $\mathcal{C}$. Notice that $u$ satisfies

$$
\Delta_{g_{0}} u-e^{2 u}=0
$$

in $A_{b}$, and in addition, letting $y$ be the polar angular variable $\theta$ on $A$, then $\Delta_{g_{0}}=\partial_{x}^{2}-(1-x)^{-1} \partial_{x}+(1-x)^{-2} \partial_{y}^{2}$ there. Hence $\Delta_{g_{0}}(\log (1 / x))-e^{2 \log (1 / x)}=$ $1 / x(1-x)$, and so $\Delta_{g_{0}} v+\left(1 / x^{2}\right)\left(1-e^{2 v}\right)=1 / x(1-x)$; we rewrite this finally as

$$
L v \equiv\left(x^{2} \Delta_{g_{0}}-2\right) v=Q(v)+r(x)
$$


where $r(x)=-x /(1-x)$ and $Q(v)=e^{2 v}-1-2 v$ is smooth and vanishes quadratically as $v \rightarrow 0$.

It may seem that we have lost ground since the linear operator $L=$ $x^{2} \Delta_{g_{0}}-2$ appearing here, while elliptic in the interior, is uniformly degenerate at $\partial \Omega$. However, this sort of degenerate elliptic operator is wellunderstood, and [14] contains a general framework for studying degenerate operators of this type. We now state the results we need from that paper and then apply them to our purposes.

We shall use a scale of weighted Hölder spaces, $x^{\gamma} \Lambda_{0}^{\ell, \alpha, \ell^{\prime}}(\bar{\Omega})$ for $\gamma \in \mathbb{R}$ and $\ell, \ell^{\prime} \in \mathbb{N}, \ell \geq \ell^{\prime}$. First, when $\gamma=0$ and $\ell^{\prime}=0$, then $\Lambda_{0}^{\ell, \alpha, 0} \equiv \Lambda_{0}^{\ell, \alpha}$ is the 'geometric Hölder space' associated to the covariant derivative for the metric $g_{1}=x^{-2} g_{0}$ (or any metric smoothly quasi-isometric to this). This mean that $w$ is in this space if the supremum over all $g_{1}$-unit balls of the Hölder seminorms with exponent $\alpha$ of the functions $\left(x \partial_{x}\right)^{j}\left(x \partial_{y}\right)^{k} w$, $j+k \leq \ell$, is finite; the norm is the obvious one. Note that all derivatives here are taken with respect to the degenerate vector fields $x \partial_{x}$ and $x \partial_{y}$. The space $\Lambda_{0}^{\ell, \alpha, \ell^{\prime}}$, still with weight parameter $\gamma=0$, consists of those elements $w \in \Lambda_{0}^{\ell, \alpha}$ such that $\partial_{y}^{k} w \in \Lambda_{0}^{\ell-k, \alpha}$ for $0 \leq k \leq \ell^{\prime}$. In other words, up to $\ell^{\prime}$ of the $x \partial_{y}$ derivatives may be replaced by derivatives with respect to the nondegenerate vector field $\partial_{y}$. Finally,

$$
x^{\gamma} \Lambda_{0}^{\ell, \alpha, \ell^{\prime}}=\left\{w=x^{\gamma} \tilde{w}: \tilde{w} \in \Lambda_{0}^{\ell, \alpha, \ell^{\prime}}\right\} .
$$

Clearly

$$
L: x^{\gamma} \Lambda_{0}^{\ell+2, \alpha, \ell^{\prime}} \rightarrow x^{\gamma} \Lambda_{0}^{\ell, \alpha, \ell^{\prime}}
$$

is bounded for every $\gamma \in \mathbb{R}$ and $0 \leq \ell^{\prime} \leq \ell$. But this map may be ill-behaved in various ways, and to understand this we must compute the indicial roots of $L$. By definition, $\gamma$ is an indicial root of $L$ if $L\left(x^{\gamma}\right)=O\left(x^{\gamma+1}\right)$. But

$$
L\left(x^{\gamma}\right)=x^{2} \Delta_{g_{0}} x^{\gamma}-2 x^{\gamma}=\left(\gamma^{2}-\gamma-2\right) x^{\gamma}+O\left(x^{\gamma+1}\right),
$$

so this can only happen if $\gamma=\gamma_{ \pm}$, where $\gamma_{-}=-1$ and $\gamma_{+}=2$. These are the only two indicial roots of $L$.

It is not hard to check that (3.9) fails to have closed range when $\gamma=\gamma_{ \pm}$. On the other hand, Corollary 6.4 and Proposition 5.30 in [14] give

Lemma 3.4 The map (3.9) is Fredholm of index zero when $-1<\gamma<2$. 
The proof relies on the construction of a parametrix $G$ for $L$ such that

$$
G: x^{\gamma} \Lambda_{0}^{\ell, \alpha, \ell^{\prime}} \longrightarrow x^{\gamma} \Lambda_{0}^{\ell+2, \alpha, \ell^{\prime}}
$$

is bounded for all $0 \leq \ell^{\prime} \leq \ell$, and such that both $G L-I$ and $L G-I$ are compact. This uses the restriction $-1<\gamma<2$, and immediately implies that (3.9) is Fredholm when $\gamma$ is in this range. The vanishing of the index follows from the formal self-adjointness of $L$ (or alternately, because $L$ is real and scalar).

To proceed further, we also need a regularity theorem, which is Proposition 3.28 in 14]:

Lemma 3.5 Suppose $-1<\gamma \leq N$ and $L w=f$, where $w \in x^{\gamma} L^{\infty}$ and $f=x^{N} \tilde{f}, \tilde{f} \in C^{\infty}(\bar{\Omega})$, then necessarily $w=x^{2} w_{1}+x^{N} w_{2}+x^{2} \log x w_{3}$ where $w_{1}, w_{2}, w_{3} \in C^{\infty}(\bar{\Omega})$. If $N>2$ here then $w_{3}=0$, i.e., the expansion for $u$ has no logarithms.

Remark 3.4. This is a specialization of a more general result which, for this operator $L$, states that if $w \in x^{\gamma} L^{\infty}, \gamma>-1$, and $L w=f$ where $f$ has a general polyhomogeneous expansion (with all exponents greater than or equal to $\gamma$ ), then $w$ also has a polyhomogeneous expansion of the same form, although possibly with terms with extra logarithmic factors.

This lemma applies immediately as follows: if $w \in x^{\gamma} \Lambda_{0}^{\ell, \alpha, \ell^{\prime}}$ with $-1<$ $\gamma<2$, and $L w=0$ then in particular, $w$ is smooth on $\bar{\Omega}$ and vanishes at $\partial \Omega$. Since solutions of $L w=0$ satisfy the maximum principle, we get $w=0$. Hence (3.9) is injective, and thus an isomorphism, when $-1<\gamma<2$.

Now recall the decomposition $u=\log (1 / x)+v$ where $|v| \leq C x$, i.e., $v \in x L^{\infty}$. Since $v$ satisfies a semilinear elliptic equation which is uniformly elliptic in unit balls relative to the metric $g_{1}=x^{-2} g_{0}$, we may use standard Schauder estimates in each of these balls, and recall the initial definition of the weighted Hölder spaces with $\ell^{\prime}=0$ to conclude that $v \in x \Lambda_{0}^{\ell, \alpha}$ for every $\ell \geq 0$.

Our next (and final) major claim is that $v \in x \Lambda_{0}^{\ell, \alpha, \ell^{\prime}}$ for every $0 \leq \ell^{\prime} \leq \ell$. Set $\gamma=1$ and let $G$ denote the corresponding inverse for $L$. Write the equation $L v=Q(v)+r(x)$ as $v=G Q(v)+G(r)$; this is legitimate because both $Q(v)$ and $r(x)$ lie in $x \Lambda_{0}^{\ell, \alpha}$ for every $\ell \geq 0$. In fact, since $r(x) \in x \Lambda_{0}^{\ell, \alpha, \ell^{\prime}}$ for every $\ell \geq \ell^{\prime}$, the boundedness of (3.11) shows that the final term is completely tangentially regular. Next, write $v=x \tilde{v}$, so that $\tilde{v} \in \Lambda_{0}^{\ell, \alpha}$. Then $Q(v)=x^{2} \tilde{Q}(x, \tilde{v})$, where $\tilde{Q}(x, s)$ again vanishes quadratically as $s \rightarrow 0$. Let us make the inductive hypothesis that $v \in x \Lambda_{0}^{\ell, \alpha, \ell^{\prime}}$ for some fixed $\ell^{\prime}$ (and 
every $\ell \geq \ell^{\prime}$ ). This is clearly true when $\ell^{\prime}=0$, so we must show that if it true for some value of $\ell^{\prime}$, then it is true when $\ell^{\prime}$ is replaced by $\ell^{\prime}+1$. This uses a commutator argument. In fact,

$$
\partial_{y} v=\partial_{y} G Q(v)+\partial_{y} G(r)
$$

Neglecting the final term on the right, which we already know has the correct regularity, reexpress the other term on the right as

$$
G\left(\partial_{y} x^{2} \tilde{Q}(\tilde{v})\right)+\left[\partial_{y}, G\right] Q(v) .
$$

By Proposition 3.30 in [14, the commutator $\left[\partial_{y}, G\right]$ enjoys the same mapping properties (3.11) as $G$ itself, and so the second term here lies in $x \Lambda^{\ell, \alpha, \ell^{\prime}}$, by the inductive hypothesis. On the other hand, $\partial_{y}\left(x^{2} \tilde{Q}(\tilde{v})\right)=\left(x \partial_{y}\right) x \tilde{Q}(\tilde{v})$, and since $x \tilde{Q}(\tilde{v}) \in x \Lambda_{0}^{\ell, \alpha, \ell^{\prime}}$, we see that this first term also has this same regularity. Thus all terms in this expression for $\partial_{y} v$ lie in $x \Lambda_{0}^{\ell, \alpha, \ell^{\prime}}$, and so $v \in x \Lambda_{0}^{\ell, \alpha, \ell^{\prime}+1}$ for all $\ell \geq \ell^{\prime}+1$. This proves the claim, and shows that $v$ is fully tangentially regular.

It remains to establish that $v=x \tilde{v}$ where $\tilde{v} \in C^{\infty}(\bar{\Omega})$. One extra consideration we need to address is that there are no logarithmic terms in the expansion for $v$, the presence of which might be suspected from Lemma 3.5. Define $\mathcal{A}^{\nu}$ to be the intersection of $x^{\nu} \Lambda_{0}^{\ell, \alpha, \ell^{\prime}}$ over all $0 \leq \ell^{\prime} \leq \ell<\infty$; we have shown that $v \in \mathcal{A}^{1}$. To deduce its expansion, write $L=x^{2} \partial_{x}^{2}-2+E$, where $E$ consists of all 'error terms' (i.e., $x^{2} \partial_{y}^{2}$ and $\left.-x^{2} /(1-x) \partial_{x}\right)$. Now regard the equation for $v$ as an ODE in $x$ with values in functions smooth on the boundary:

$$
x^{2} \partial_{x}^{2} v-2 v=-E v+Q(v)+r(x) .
$$

We think of the whole right hand side as an inhomogeneous term. Recall that $r(x)=-x /(1-x)=-x-x^{2}-\ldots$, and $Q(v)=e^{2 v}-1-2 v=2 v^{2}+O\left(v^{3}\right)$. Then at the first stage the right hand side has the form $-x+f_{2}$, with $f_{2} \in \mathcal{A}^{2}$. Integrating the ODE gives $v=-(1 / 2) x+v_{2}, v_{2} \in \mathcal{A}^{2}$. Inserting this into the right side shows that the sum of these terms on the right have the form $-x+f_{3}, f_{3} \in \mathcal{A}^{3}$. The fact that the $x^{2}$ term in this expansion vanishes is a special feature, due to a fortuitous cancellation; the absence of this term is what precludes the logarithm terms in the expansion for $v$. Integrating the ODE again shows that $v=-(1 / 2) x+v_{2}(y) x^{2}+v_{3}$, where $v_{3} \in \mathcal{A}^{3}$ and $v_{2}(y) \in C^{\infty}(\partial \Omega)$. Inserting this back into the right side and iterating this argument gives the complete expansion for $v$. This completes the proof. 
Remark 3.5. From (3.3) one can compute $\left.\partial_{\nu}^{2} W\right|_{\partial \Omega}$, and see that in the expansion (3.1) $u_{1}(y)=\kappa(y) / 2$, where $\kappa(y)$ is the curvature of $\partial \Omega$ at $y$. On the other hand, the coefficient $u_{2}(y)$ depends on the global behavior of $\Omega$, as one can see by examining (1.7) for different values of $b$.

\section{General planar domains}

In this section we construct Poincaré metrics on general planar domains, as long as the complement contains at least two points. To begin, given $\Omega \subset \mathbb{R}^{2}$, open and connected, take a sequence $\Omega_{\nu}$ bounded, with smooth boundary, such that $\Omega_{\nu} \subset \subset \Omega_{\nu+1}$ and $\Omega_{\nu} \nearrow \Omega$, in the sense that any compact $K \subset \Omega$ is contained in $\Omega_{\nu}$ for large $\nu$. Let $u_{\nu}$ be the solutions to (1.2) on $\Omega_{\nu}$ such that $\left.u_{\nu}\right|_{\partial \Omega_{\nu}}=+\infty$ and $g_{j k}^{\nu}=e^{2 u_{\nu}} \delta_{j k}$ are complete metric tensors on $\Omega_{\nu}$, as in Proposition 2.4 (obtained as in (2.9)). The argument used to prove Lemma 2.1 shows that $u_{\nu} \searrow$ as $\nu \nearrow \infty$. Our main goal in this section is to establish the following.

Proposition 4.1 If $\Omega \subset \mathbb{R}^{2}$ is a connected open set with the property that $\mathbb{R}^{2} \backslash \Omega$ contains at least two points, then

$$
u_{\nu} \searrow u \text { as } \nu \nearrow \infty
$$

where $u \in C^{\infty}(\Omega)$, solving (1.2), and the metric tensor $g_{j k}=e^{2 u} \delta_{j k}$ is a complete metric tensor on $\Omega$, of Gauss curvature -1 .

As a warm-up, we first give a simple proof of the following special case, which extends Proposition 2.4, in the case of planar domains.

Proposition 4.2 Proposition 4.] holds when $\Omega \subset \mathbb{R}^{2}$ is a bounded, connected, open set, whose boundary satisfies the following regularity hypothesis:

Each $p \in \partial \Omega$ is the endpoint of a line segment in $\mathbb{R}^{2} \backslash \Omega$.

Proof: First we need to get a bound on $u_{\nu}$ from below. Indeed, taking $\Omega$ inside a sufficiently large disk $D_{b}(0)$, with Poincaré metric $e^{2 w} \delta_{j k}$, then $u_{\nu} \geq w$ on $\Omega_{\nu}$. This gives a locally bounded $u$ on $\Omega$ which satisfies (4.1). As before elliptic estimates give smooth convergence to $u \in C^{\infty}(\Omega)$, solving (1.2). 
Completeness remains to be demonstrated. Under the hypothesis (4.2), the completeness proof goes as follows. Say $\gamma:[0, L) \rightarrow \Omega$ is a unit-speed geodesic (for $g_{j k}$ ) and suppose $L<\infty$ and $\gamma(t)$ does not converge to a point in $\Omega$ as $t \rightarrow L$. As in the proof of Proposition 2.3, we have $\gamma(t) \rightarrow p$ for some $p \in \partial \Omega$.

Let $\ell$ be a line segment in $\mathbb{R}^{2} \backslash \Omega$ with $p$ as an endpoint. Regard $\mathbb{R}^{2} \subset \widehat{\mathbb{C}}$. Now it is elementary to produce a conformal diffeomorphism $\psi: \widehat{\mathbb{C}} \backslash \ell \rightarrow D_{1}$; pull back the Poincaré metric on $D_{1}$ to get a complete metric $e^{2 w} \delta_{j k}$ on $\widehat{\mathbb{C}} \backslash \ell$, of Gauss curvature -1 . Again we have $u_{\nu} \geq w$ on $\Omega_{\nu}$, for each $\nu<\infty$, and hence $u \geq w$ on $\Omega$, and the completeness of $e^{2 u} \delta_{j k}$ on $\Omega$ is proven.

The proof of Proposition 4.1 for more general $\Omega$ requires more work, which we now undertake. To get a lower bound on $u_{\nu}$ this time, we make use of the following result.

Lemma 4.3 The region $\mathbb{C} \backslash\{0,1\}$ has a Poincaré metric.

We will give a curvature equation proof of this lemma after we apply it to prove Proposition 4.1.

Returning to the estimation of $u_{\nu}$ in the proof of Proposition 4.1, say $p_{1}, p_{2} \in \mathbb{R}^{2} \backslash \Omega$. Lemma 4.3 also holds for $\mathbb{R}^{2} \backslash\left\{p_{1}, p_{2}\right\}$, which therefore has a Poincaré metric

$$
h_{j k}=e^{2 w} \delta_{j k} .
$$

Now as in Lemma 2.1 we have $u_{\nu} \geq w$ on $\Omega_{\nu}$. Hence, as before we can deduce that $u_{\nu} \searrow u$ with $u \in C^{\infty}(\Omega)$ satisfying (1.1). To prove that the metric $e^{2 u} \delta_{j k}$ is complete on $\Omega$, we argue as before that if not, there would exist a unit-speed $\gamma:[0, L) \rightarrow \Omega$ with $\gamma(t) \rightarrow p \in \partial \Omega$ as $t \rightarrow L$. Here $\partial \Omega$ denotes the boundary of $\Omega$ in $\widehat{\mathbb{C}}$, so either $p \in \mathbb{R}^{2} \backslash \Omega$ or $p=\infty$. We now bring in the metric (4.3), with $p_{j} \in \mathbb{R}^{2} \backslash \Omega$ and with $p_{1}=p$ if $p \neq \infty$; using $u \geq w$ we again have that $e^{2 u} \delta_{j k}$ is complete on $\Omega$. This gives Proposition 4.1. modulo a proof of Lemma 4.3.

We turn now to a proof of Lemma 4.3. One ingredient will be a metric on $\mathbb{C} \backslash\{0,1\}$ of the form $e^{2 w_{0}(z)} \delta_{j k}$, with

$$
e^{w_{0}}=A \frac{\left(1+r^{a}\right)^{b}}{r^{c}} \frac{\left(1+\rho^{a}\right)^{b}}{\rho^{c}}, \quad r=|z|, \rho=|z-1|,
$$

with $A, a, b, c>0$. A calculation of the Gauss curvature for this metric gives

$$
K=-\frac{a^{2} b}{A^{2}}\left[\frac{r^{a-2+2 c} \rho^{2 c}}{\left(1+r^{a}\right)^{2+2 b}\left(1+\rho^{a}\right)^{2 b}}+\frac{r^{2 c} \rho^{a-2+2 c}}{\left(1+r^{a}\right)^{2 b}\left(1+\rho^{a}\right)^{2+2 b}}\right] .
$$


We have $K<0$ and it is bounded away from zero as long as

$$
a-2+2 c \leq 0, \quad 4 c-a-4 a b-2 \geq 0 .
$$

For example, we can take

$$
a=\frac{1}{3}, \quad b=\frac{1}{2}, \quad c=\frac{5}{6},
$$

the parameters used in [13], pp. 78-80. If $A>0$ is small enough, we have $K \leq-1$.

Fix such $A$, let $\Omega_{\nu} \nearrow \Omega=\mathbb{C} \backslash\{0,1\}$, and take $u_{\nu} \in C^{\infty}\left(\Omega_{\nu}\right)$, as in Proposition 4.1, with $u_{\nu} \searrow$ as $\nu \nearrow \infty$. A variant of the proof of Lemma 2.1 gives $u_{\nu} \geq w_{0}$ on $\Omega_{\nu}$, with $w_{0}$ given by (4.4), so we have convergence: $u_{\nu} \rightarrow u$ with $u \in C^{\infty}(\Omega)$ satisfying (1.2) and $u \geq w_{0}$ on $\Omega$. However, the metric $e^{2 w_{0}} \delta_{j k}$ is not complete, so we need to do some more work to show that $e^{2 u} \delta_{j k}$ is complete on $\mathbb{C} \backslash\{0,1\}$.

To check completeness of $e^{2 u} \delta_{j k}$ near 0 , we compare it with the metric (1.5), i.e., $e^{2 v} \delta_{j k}$, where

$$
e^{2 v}=\left(r \log \frac{1}{r}\right)^{-2}
$$

on $0<r<1$. Given the convergence $u_{\nu} \rightarrow u$, we can find a constant $B \geq 0$ such that $u_{\nu} \geq v-B$ on $\{z \in \mathbb{C}:|z|=1 / 2\}$. Note that $e^{2(v-B)} \delta_{j k}$ has curvature $-e^{2 B} \leq-1$ on $D^{*}$. Now a variant of Lemma 2.1 gives $u_{\nu} \geq v-B$ on $\left\{z \in \Omega_{\nu}:|z| \leq 1 / 2\right\}$, and hence

$$
u \geq v-B \text { on }\{z: 0<|z| \leq 1 / 2\} .
$$

This implies completeness of $e^{2 u} \delta_{j k}$ near 0 . Completeness near 1 is established similarly. The formula (1.5) also defines a Poincaré metric on $\{z:|z|>1\}$, and this can be used to show that $e^{2 u} \delta_{j k}$ is complete near $\infty$. Lemma 4.3 is proven.

Remark. Lemma 4.3 is equivalent to the assertion that there is a holomorphic covering map

$$
\psi: D_{1} \longrightarrow \mathbb{C} \backslash\{0,1\}
$$

This result is an ingredient in the classical theorems of Picard. The map $\psi$ can be constructed explicitly via elliptic function theory. Cf. Chapter 7 of [1]; this provided the original proof. This covering can also be constructed by 
applying Schwarz reflection to the Riemann mapping function of a special domain on $\mathbb{C}$ (cf. Chapter 5, $\S 6$ of [20]). A variant of (4.4), obtained by adding multiples of (1.5) and its images near 0,1 , and $\infty$, was produced in [9] and shown there to have Gauss curvature $\leq-1$ and to be complete on $\mathbb{C} \backslash\{0,1\} ;$ cf. 12, pp. $7-10$.

We can produce other Riemann surfaces covered by the disk, using the following simple result.

Proposition 4.4 If $M$ is a Riemann surface with a holomorphic covering map $\psi: D_{1} \rightarrow M$ and $\Omega \subset M$ is a nonempty open connected set, then there exists a holomorphic covering map $\varphi: D_{1} \rightarrow \Omega$.

Proof: If $\mathcal{O} \subset D_{1}$ is a connected component of $\psi^{-1}(\Omega)$, then $\psi$ restricts to a holomorphic covering $\psi: \mathcal{O} \rightarrow \Omega$. By Proposition 4.2, there exists a holomorphic covering $\tilde{\psi}: D_{1} \rightarrow \mathcal{O}$. Composing gives the holomorphic covering $\varphi: D_{1} \rightarrow \Omega$.

We will not dwell on applications of this last proposition, since they would all be subsumed by the results of $\S 6$.

\section{$5 \quad$ Koebe's disk theorem}

Here we make note of a simple curvature proof of some results of P. Koebe on the family $\mathcal{S}$ of univalent (i.e., one-to-one) holomorphic maps $f: D_{1} \rightarrow \mathbb{C}$ satisfying $f(0)=0, f^{\prime}(0)=1$. Here is the first result.

Proposition 5.1 There exists a constant $b \in(1, \infty)$ such that for any $f \in$ $\mathcal{S}, \Omega=f\left(D_{1}\right)$ has the property

$$
\frac{1}{b} \leq \operatorname{dist}(0, \partial \Omega) \leq 1
$$

Here (and in (5.2), (5.4) below) we use Euclidean distance, so that $\operatorname{dist}(0, \partial \Omega)=\inf \{|z|: z \in \partial \Omega\}$. L. Bieberbach showed that one can take $b=4$, and this is sharp. This sharpened version of Proposition 5.1 is called the Koebe-Bieberbach quarter theorem. Our method does not yield $b=4$. The following result is equivalent to Proposition 5.1. 
Proposition 5.2 Let $\Omega$ be a proper, simply connected domain in $\mathbb{C}$. Let $e^{2 u} \delta_{j k}$ be the Poincaré metric on $\Omega$. Then, for all $p \in \Omega$,

$$
\frac{1}{2} \operatorname{dist}(p, \partial \Omega) \leq e^{-u(p)} \leq \frac{b}{2} \operatorname{dist}(p, \partial \Omega)
$$

To see the equivalence, note that if $f: D_{1} \rightarrow \Omega$ is biholomorphic and $\gamma(z)^{2}|d z|^{2}$ is a metric on $\Omega$, then $D_{1}$ inherits the metric $\gamma(f(z))^{2}\left|f^{\prime}(z)\right|^{2}|d z|^{2}$. Thus the Poincaré metric $e^{2 u} \delta_{j k}$ induced on $\Omega$ has

$$
e^{-u(f(z))}=\frac{1}{2}\left(1-|z|^{2}\right)\left|f^{\prime}(z)\right|
$$

Picking a biholomorphic $f$ such that $f(0)=p$ yields the equivalence of these propositions easily. In addition, comparing (5.2) and (5.3) gives the following result.

Proposition 5.3 If $f: D_{1} \rightarrow \Omega$ is a biholomorphic map, then, for all $z \in D_{1}$,

$$
\operatorname{dist}(f(z), \partial \Omega) \leq\left(1-|z|^{2}\right)\left|f^{\prime}(z)\right| \leq b \operatorname{dist}(f(z), \partial \Omega) .
$$

We note that the upper estimate of $\operatorname{dist}(0, \partial \Omega)$ in (5.1) and (equivalently) the lower estimate on $e^{-u(p)}$ in (5.2) are elementary. In fact, the lower estimate in (5.2) has already been given in (2.6); alternatively the upper estimate in (5.1) follows from the Schwarz lemma.

It remains to prove the upper estimate on $e^{-u(p)}$ in (5.2), and we turn to that task. Note that a dilation of $\mathbb{C}$ multiplies all quantities in (5.2) by the same factor, so there is no loss of generality in assuming $\operatorname{dist}(p, \partial \Omega)=1 / 2$. Say $q_{1} \in \partial \Omega,\left|p-q_{1}\right|=1 / 2$. Note that $\mathbb{C} \backslash \Omega$ is connected and not bounded, so there exists $q_{2} \in \mathbb{C} \backslash \Omega$ such that $\left|q_{1}-q_{2}\right|=1$. Now, as noted in the proof of Proposition 4.1, we have $e^{2 u} \geq e^{2 w}$ on $\Omega$, where $e^{2 w} \delta_{j k}$ is the Poincaré metric on $\mathbb{C} \backslash\left\{q_{1}, q_{2}\right\}$. In view of the obvious relation between the Poincaré metric on $\mathbb{C} \backslash\left\{q_{1}, q_{2}\right\}$ and the Poincaré metric (call it $\Phi^{2} \delta_{j k}$ ) on $\mathbb{C} \backslash\{0,1\}$, we have

$$
\operatorname{dist}(p, \partial \Omega)=\frac{1}{2} \Longrightarrow e^{u(p)} \geq \inf _{|z|=1 / 2} \Phi(z)>0,
$$

and the proof is complete. 
Remark. The simple argument above reveals the role of the simple connectivity of $\Omega$ in the estimate on the Poincaré metric. In [- 1 ] there is a more sophisticated argument yielding an estimate on the Poincaré metric of planar domains that are not simply connected.

One classical use of Koebe's disk theorem is to provide a uniform bound on $|f(z)|$ for $f \in \mathcal{S}$. We record a short derivation of such a bound from (5.4). To begin, the second inequality in (5.4) implies $\left(1-|z|^{2}\right)\left|f^{\prime}(z)\right| \leq$ $b(1+|f(z)|)$. Noting that $f\left(D_{1 / 2}\right) \supset D_{1 / 2 b}$, we see that, for all $f \in \mathcal{S}$,

$$
|z| \geq \frac{1}{2} \Rightarrow|f(z)| \geq \frac{1}{2 b} \Rightarrow\left|\frac{f^{\prime}(z)}{f(z)}\right| \leq \frac{B}{1-|z|^{2}}, \quad B=b(1+2 b) .
$$

Since $f\left(D_{1 / 2}\right)$ does not contain $\bar{D}_{1 / 2}$, we see that there exists $z(f)$ such that $|z(f)|=1 / 2$ and $|f(z(f))|=1 / 2$. Using (5.6) and integrating over an arc of $\{z:|z|=1 / 2\}$, we have an absolute bound

$$
|z|=\frac{1}{2} \Longrightarrow|f(z)| \leq C, \quad \forall f \in \mathcal{S} .
$$

This bound also holds for $|z| \leq 1 / 2$. Then radial integration of (5.6) yields an absolute bound

$$
|f(z)| \leq C_{2}(1-|z|)^{-B / 2}, \quad \forall f \in \mathcal{S} .
$$

There exist sharper bounds on elements of $\mathcal{S}$, obtained by harder work; cf. [2], p. 84. However, the bound derived above suffices for the following normal family result, also due to Koebe. We record the essentially standard proof.

Proposition 5.4 The set $\mathcal{S}$ is compact in $\mathcal{H}\left(D_{1}\right)$, the space of holomorphic functions on $D_{1}$.

Proof: Take $f_{\nu} \in \mathcal{S}$. The uniform bounds $\left|f_{\nu}(z)\right| \leq K(r)$ for $|z| \leq r$ established above imply some subsequence converges locally uniformly to $f \in \mathcal{H}\left(D_{1}\right)$. We have $f(0)=0$ and $f^{\prime}(0)=1$. That $f$ is univalent is then a simple consequence of Hurwitz' theorem.

Remark. A direct proof of Proposition 5.4, not using Proposition 5.1, but somewhat longer and trickier, is given in [8]]. 


\section{The uniformization theorem}

In this section we prove the uniformization theorem for general noncompact Riemann surfaces:

Theorem 6.1 If $M$ is a noncompact, simply connected Riemann surface, then $M$ is holomorphically equivalent to either $D_{1}$ or $\mathbb{C}$.

To prove this, we begin by taking $\Omega_{\nu} \subset \subset \Omega_{\nu+1} \nearrow M$, such that each set $\bar{\Omega}_{\nu}$ is compact, with smooth boundary, and simply connected. (This relies on some results on the topology of surfaces, such as the a priori knowledge that $M$ is diffeomorphic to $D_{1}$ ). Our argument from here parallels one in [8], except that we apply the method of the curvature equation to each $\bar{\Omega}_{\nu}$.

In detail, say $p \in \Omega_{0} \subset \subset \Omega_{1} \subset \subset \cdots$. By Proposition 2.4 we have for each $\nu$ a holomorphic diffeomorphism

$$
\psi_{\nu}: \Omega_{\nu} \longrightarrow D_{1}, \quad \psi_{\nu}(p)=0 .
$$

Take $\alpha_{\nu}=D \psi_{\nu}(p) \in \operatorname{Hom}\left(T_{p} M, \mathbb{C}\right)$. Then $\alpha_{\nu}=a_{\nu} \alpha_{0}$ for uniquely defined $a_{\nu} \in \mathbb{C}$, and if we set

$$
\varphi_{\nu}: \Omega_{\nu} \longrightarrow D_{R_{\nu}}, \quad R_{\nu}=\left|a_{\nu}\right|^{-1}, \quad \varphi_{\nu}(x)=a_{\nu}^{-1} \psi_{\nu}(x),
$$

we have

$$
D \varphi_{\nu}(p)=D \varphi_{0}(p)=\alpha_{0}, \quad \forall \nu .
$$

It follows from the Schwarz lemma that $\left|a_{0}\right|>\left|a_{1}\right|>\left|a_{2}\right|>\cdots$, and hence

$$
R_{0}<R_{1}<R_{2}<\cdots \text {. }
$$

To proceed, let us consider

$$
\Phi_{\nu}=\varphi_{\nu} \circ \varphi_{0}^{-1}: D_{1} \longrightarrow D_{R_{\nu}} .
$$

We have each $\Phi_{\nu}$ holomorphic and one-to-one (i.e., univalent), and

$$
\Phi_{\nu}(0)=0, \quad \Phi_{\nu}^{\prime}(0)=1 .
$$

At this point we apply Koebe's normal family theorem, established in the last section. Thus we see that a subsequence of $\left.\varphi_{\nu}\right|_{\Omega_{0}}$ converges to a univalent map $\Omega_{0} \rightarrow \mathbb{C}$. A similar consideration of

$$
\Phi_{\nu \mu}=\varphi_{\nu} \circ \varphi_{\mu}^{-1}: D_{R_{\mu}} \longrightarrow D_{R_{\nu}}, \quad \nu \geq \mu,
$$


plus a diagonal argument yields a subsequence of $\left(\varphi_{\nu}\right)$ converging to a univalent holomorphic map

$$
\varphi: M \longrightarrow \mathbb{C}
$$

From here one could argue that $\varphi$ maps $M$ biholomorphically onto $D_{R}$, where $R$ is the supremum of the sequence (6.4); cf. [8]. For our purposes we can bypass this argument. At this point we have $M$ holomorphically equivalent to $\Omega=\varphi(M) \subset \mathbb{C}$, and the results of $\S 4$ are applicable, to show that either $\Omega=\mathbb{C}$ or $\Omega$ is holomorphically equivalent to $D_{1}$.

\section{The curvature dichotomy}

Let $\Omega$ be a noncompact Riemann surface, with a compatible Riemannian metric $g_{0}$. Take compact, smoothly bounded $\bar{\Omega}_{\nu} \subset \Omega$ such that $\Omega_{\nu} \nearrow \Omega$, and let $u_{\nu} \in C^{\infty}\left(\Omega_{\nu}\right)$ be solutions to the curvature equation (1.2) such that $e^{2 u_{\nu}} g_{0}$ is a Poincaré metric on $\Omega_{\nu}$. As we have seen, $u_{\nu} \searrow$ as $\nu \nearrow \infty$. We tackle the issue of convergence of $u_{\nu}$.

Proposition 7.1 For each $\Omega$ one of the following must happen:

1) $u_{\nu} \searrow u \in C^{\infty}(\Omega)$, where $u$ satisfies (1.8), or

2) $u_{\nu} \searrow-\infty$ on $\Omega$.

In case 2), $\Omega$ has no metric conformal to $g_{0}$ with Gauss curvature $\leq-1$.

Proof: We already know that if all $u_{\nu} \geq v$ with $v$ locally bounded then case (1) holds. Suppose $u_{\nu}\left(p_{\nu}\right) \rightarrow-\infty$ for some sequence $p_{\nu} \in \mathcal{O} \subset \subset \Omega_{N}$; from now on we take $\nu \geq N+1$. Since $u_{\nu} \leq u_{N+1}$ for $\nu \geq N+1$, we have a uniform upper bound on $\bar{\Omega}_{N}$ : $u_{\nu} \leq A_{N}<\infty$ for $\nu \geq N+1$. We thus have a bound

$$
\left|e^{2 u_{\nu}}+k\right| \leq A_{2 N} \quad \text { on } \quad \bar{\Omega}_{N}, \quad \nu \geq N+1
$$

Hence we can find $v_{\nu} \in C^{1}\left(\bar{\Omega}_{N}\right)$, for $\nu \geq N+1$, such that

$$
\Delta v_{\nu}=e^{2 u_{\nu}}+k \text { on } \bar{\Omega}_{N}, \quad\left\|v_{\nu}\right\|_{L^{\infty}\left(\Omega_{N}\right)} \leq A_{3 N} .
$$

Hence

$$
\Delta\left(u_{\nu}-v_{\nu}\right)=0 \text { on } \Omega_{N}, \quad-\infty<u_{\nu}-v_{\nu} \leq A_{4 N}
$$


for $\nu \geq N+1$. That $u_{\nu}\left(p_{\nu}\right) \rightarrow-\infty$ implies $u_{\nu} \rightarrow-\infty$ on $\overline{\mathcal{O}}$ now follows from Harnack's estimate.

As for the last assertion of Proposition 7.1, note that if $e^{2 w} g_{0}$ has Gauss curvature $\leq-1$ on $\Omega$ then $u_{\nu} \geq w$ for all $\nu$.

Making use of Theorem 6.1, we can restate the dichotomy in Proposition 7.1 as follows.

Proposition 7.2 In the setting of Proposition 7.1, case (1) holds if and only if $D_{1}$ covers $\Omega$ and case (2) holds if and only if $\mathbb{C}$ covers $\Omega$.

Proof: Suppose case (1) holds; we show $\Omega$ cannot be covered by $\mathbb{C}$. Indeed, a holomorphic covering $f: \mathbb{C} \rightarrow \Omega$ would pull back the metric $e^{2 u} g_{0}$ to a metric on $\mathbb{C}$ of curvature -1 . However, as one sees by looking at the metrics (2.7) on the disks $D_{\beta}$ and letting $\beta \nearrow \infty$, case (2) holds for $\mathbb{C}$, so this is not possible.

On the other hand, suppose case (2) holds; we claim $\Omega$ cannot be covered by $D_{1}$. Indeed, a holomorphic covering $f: D_{1} \rightarrow \Omega$ puts a Poincaré metric on $\Omega$, which we have seen cannot hold in case (2).

Since case (1) yields a holomorphic covering $f: D_{1} \rightarrow \Omega$, which puts a Poincaré metric $e^{2 v} g_{0}$ on $\Omega$, and since $u_{\nu} \geq v$ on $\Omega_{\nu}$, we have $u \geq v$, and hence $u=v$, so:

Corollary 7.3 In case (1), the limit $u$ yields a Poincaré metric $e^{2 u} g_{0}$ on $\Omega$. In particular, whenever $\Omega$ has a metric of curvature $\leq-1$, it has a conformally equivalent Poincaré metric.

When $\Omega$ is simply connected, the dichotomy in Proposition 7.1 is precisely the dichotomy between hyperbolic and parabolic Riemann surfaces, defined as follows.

Definition. A noncompact (connected) Riemann surface $\Omega$ is hyperbolic if and only if there exists a nonconstant, nonpositive, subharmonic function on $\Omega$, and parabolic otherwise.

The proof of the uniformization theorem found in most sources (for example, [2], [7], [19]) proceeds by separately treating these two cases. It is shown that a simply connected hyperbolic Riemann surface is equivalent to $D_{1}$ and a simply connected parabolic Riemann surface is equivalent to $\mathbb{C}$. In these treatments, the first step in the hyperbolic case is the construction of a negative Green function $u$, harmonic on $\Omega \backslash\{p\}$ and behaving like log $|x|$ 
in local normal coordinates centered at $p$. Such a function has a harmonic conjugate $v$, well defined $\bmod 2 \pi \mathbb{Z}$, on $\Omega \backslash\{p\}$, and the function

$$
f(x)=e^{u(x)+i v(x)}
$$

yields a holomorphic map $f: \Omega \rightarrow D_{1}$. It is then shown that this map is a holomorphic diffeomorphism. This argument is highly nontrivial, much more subtle than the demonstration of the analogue in the context of (2.17). However, 10] produced an ingenious demonstration, used in most modern treatments. A short argument to prove the parabolic case is given in [17], and other proofs can be found in the references cited above. By contrast, the proof given in $\S 6$ of this paper is in some respects closer to Koebe's original demonstration; compare the treatment in [21], pp. 421-422.

\section{Compact Riemann surfaces}

Here we discuss the uniformization theorem for compact Riemann surfaces, given by the following classical result.

Proposition 8.1 Let $M$ be a compact, connected Riemann surface, with Euler characteristic $\chi(M)$.

1) If $\chi(M)=2$, then $M$ is holomorphically equivalent to the Riemann sphere.

2) If $\chi(M)=0$, then $M$ is holomorphically equivalent to a flat torus $\mathbb{T}_{\Lambda}=\mathbb{C} / \Lambda$.

3) If $\chi(M)<0$, then $M$ is holomorphically covered by $D_{1}$.

In cases 2) and 3), the universal cover $\widetilde{M}$ of $M$ is noncompact and Theorem 6.1 applies. If we allow ourselves to use the topological classification of surfaces, we know that in case 3) the group of covering transformations of $\widetilde{M} \rightarrow M$ is noncommutative, so there is a noncommutative discrete group of holomorphic automorphisms of $\widetilde{M}$, acting with no fixed points. A holomorphic automorphism of $\mathbb{C}$ without fixed points must be a translation, and all translations commute, so in this case $\widetilde{M}$ must be conformally equivalent to $D_{1}$. On the other hand, in case 2) the group of covering transformations of $\widetilde{M} \rightarrow M$ is isomorphic to $\mathbb{Z}^{2}$. One can check that there is no group of fixed-point-free holomorphic automorphisms of $D_{1}$ isomorphic to $\mathbb{Z}^{2}$. 
Case 1) is often proved via the Riemann-Roch theorem, which implies that, given $p \in M$, the space of meromorphic functions with at most one simple pole, at $p$, has dimension 2 , provided $\chi(M)=2$. This space includes the constant functions, but it must also contain a meromorphic function $f$ on $M$ with one simple pole (at $p$ ). Then $f$ defines a holomorphic map $f: M \rightarrow \widehat{\mathbb{C}}$. We see that $f$ has degree one, and it follows that $f$ is a holomorphic diffeomorphism.

In fact, all cases of Proposition 8.1 have PDE proofs where one starts with some Riemannian metric on $M$ compatible with the given conformal structure. We mention PDE treatments of these three cases.

To treat case 1 ), we note that given a distribution $\delta^{\prime}$ of order 1 supported at $p \in M$ such that $\left\langle 1, \delta^{\prime}\right\rangle=0$ (a derivative of a delta function) we can solve $\Delta u=\delta^{\prime}$. This can be done on any compact, connected $M$, but in case 1 ) we can say that $M \backslash\{p\}$ is simply connected. Hence $u$ is the real part of a meromorphic function $f$ on $M$ with one simple pole (at $p$ ), and as noted in our previous discussion of case 1), this yields a holomorphic diffeomorphism $f: M \rightarrow \widehat{\mathbb{C}}$.

To treat case 2 ), we can solve the curvature equation, which in this case is $\Delta u=K_{0}(x)$. Note that by the Gauss-Bonnet theorem $\int_{M} K_{0} d A=0$, so we can solve for $u$. Hence $M$ has a conformally equivalent flat metric, from which the conclusion follows.

Case 3) can be established by solving the curvature equation (1.2). This was accomplished by [5]; expositions can also be found in [3] and in Chapter 14 of [20]. Here we merely mention that the solution to the curvature equation (1.2) minimizes

$$
F(u)=\int_{M}\left(\frac{1}{2}|d u|^{2}+K_{0}(x) u\right) d A
$$

on the set

$$
S=\left\{u \in H^{1}(M): \int_{M} e^{2 u} d A=-2 \pi \chi(M)\right\} .
$$

There are other very interesting analytic avenues to uniformization in the compact case; these include the use of the determinant of the Laplacian [16] and a fourth-order heat flow [6].

\section{References}

[1] L. Ahlfors, Complex Analysis, McGraw-Hill, New York, 1966. 
[2] L. Ahlfors, Conformal Invariants, McGraw-Hill, New York, 1973.

[3] T. Aubin, Nonlinear Analysis on Manifolds. Monge-Ampere Equations, Springer-Verlag, New York, 1982.

[4] A. Beardon and C. Pommerenke, The Poincaré metric of plane domains, J. London Math. Soc. 18 (1979), 475-483.

[5] M. Berger, On Riemannian structures of prescribed Gaussian curvature for compact 2-manifolds, J. Diff. Geom. 5 (1971), 328-332.

[6] P. Chrusciel, Semi-global existence and convergence of solutions of the Robinson-Trautman (2-dimensional Calabi) equation, Commun. Math. Phys. 137 (1991), 289-313.

[7] H. Farkas and I. Kra, Riemann Surfaces, Second edition, SpringerVerlag, New York,

[8] O. Forster, Lectures on Riemann Surfaces, Springer-Verlag, New York, 1981.

[9] H. Grauert and H. Reckziegel, Hermitesch Metriken und normale Familien holomorpher Abbildunen, Math. Zeit. 89 (1965), 108-125.

[10] M. Heinz, The conformal mapping of simply connected Riemann surfaces, Ann. Math. 50 (1949), 686-690.

[11] J. Kazdan and F. Warner, Curvature functions for compact 2manifolds, Ann. of Math. 99 (1974), 14-47.

[12] S. Kobayashi, Hyperbolic Manifolds and Holomorphic Mappings, M. Dekker, New York, 1970.

[13] S. Krantz, Complex Analysis: the Geometric Viewpoint, Carus Math. Ser. \#23, MAA, 1990.

[14] R. Mazzeo, Elliptic theory of differential edge operators I, Comm. PDE 16 (1991), 1615-1664.

[15] R. Mazzeo, Regularity for the singular Yamabe problem, Indiana Univ. Math. J. 40 (1991), 1277-1299.

[16] B. Osgood, R. Phillips and P. Sarnak, Extremals of determinants of Laplacians, J. Funct. Anal. 80 (1988), 212-234. 
[17] R. Osserman, Koebe's general uniformization theorem: the parabolic case, Ann. Acad. Scient. Fennicæ Series A I, no. 258 (1958), 1-7.

[18] E. Picard, De l'equation $\Delta u=k e^{u}$ sur une surface de Riemann fermée, J. de Math. (4) 9 (1893), 273-291.

[19] G. Springer, Introduction to Riemann Surfaces, Addison-Wesley, Reading, Mass., 1957.

[20] M. Taylor, Partial Differential Equations, Vols. 1-3, Springer-Verlag, New York, 1996.

[21] M. Tsuji, Potential theory and Modern Function Theory, Chelsea, New York, 1959. 